\title{
Birefringence of mechanically clamped TGS crystals with L-valine admixture
}

\author{
Vasyl STADNYK $^{1 *}$, Mykola ROMANYUK ${ }^{1}$, Yurij KIRYK ${ }^{1}$, Ruslan BREZVIN ${ }^{1}$ \\ ${ }^{1}$ Faculty of Physics, Ivan Franko National University of Lviv, Kyryla i Mefodiya St. 8, 79005 Lviv, Ukraine \\ * Corresponding author. E-mail: vasylstadnyk@ukr.net
}

Received June 15, 2010; accepted May 18, 2011; available on-line November 8, 2011

The influence of uniaxial mechanical stresses applied along the crystal-physical axes on the principal values of the birefringence, $\Delta n_{i}$, was studied for TGS crystals with $5 \%$ L-valine admixture. It was found that $\Delta n_{i}$ are quite sensitive to stress and adopt different values and opposite signs, when compared to pure TGS. The baric coefficients $\partial T_{c} / \partial \sigma_{m}$ for the phase transition (PT) temperature appear to be less than for pure TGS. The temperature dependence of the combined piezooptic constants $\pi_{i m}^{0}$ was calculated. The values of the jumplike changes occurring for all the piezooptic constants at the PT are notably lower for the doped crystals than for pure TGS.

\section{Birefringence / Uniaxial pressure / Phase transitions / Piezooptic constants}

\section{Introduction}

Triglycine sulphate $\left(\mathrm{NH}_{2} \mathrm{CH}_{2} \mathrm{COOH}\right)_{3} \cdot \mathrm{H}_{2} \mathrm{SO}_{4}$ (TGS) represents one of the isomorphic ferroelectric molecular compounds of glycine with inorganic acids. Introduction of organic admixtures into the TGS crystal promotes fixing of the polarization $+P_{s}$ or $-P_{s}$ and appearance of the biasing field $E_{b}$ [1]. Due to this, the dielectric parameters of the doped crystals change during the aging process, a fact that should be properly accounted for when studying and exploring such crystals. The effect of L- and D-alanine admixtures on different physical properties of TGS has been studied earlier [2-5].

Differently from pure TGS, in the case of TGS crystals doped with L-valine (LVTGS) (110) facets are absent, and the (111) and (100) ones grow only at low dopant concentrations [6]. A temporal change of the domain structure, taking place during spontaneous aging, has been observed in doped TGS crystals: the number of domains decreases and the domains become much larger.

LVTGS is characterized by biasing fields, which may be used for determining the dopant concentration. These fields affect the properties of the crystal the same way as external fields applied to the crystal, so that the sample becomes spontaneously single-domain or a particular geometry of the domain structure is kept fixed [7,8]. Introduction of L-threonine and L-valine leads to weakening temperature dependence of the refractive indices, birefringence and thermal expansion of the crystals $[9,10]$.
Anomalies of the piezooptic constants near the phase transition (PT) have been revealed in pure TGS crystals and their isomorphic substituted analogues (in the inorganic part of the chemical formula, i.e. $\mathrm{SO}_{4}{ }^{2-}$, $\mathrm{SeO}_{4}{ }^{2-}$ and $\mathrm{BeF}_{4}{ }^{2-}$ ) [11-15].

In this work we study the influence of L-valine admixtures $\left(\mathrm{CH}_{3}\right)_{2}-\mathrm{CH}-\mathrm{CHNH}_{2} \mathrm{COOH}$ on the birefringent properties of TGS crystals.

\section{Experimental}

The temperature and spectral dependences of the birefringence were measured by the interference technique. Uniaxial mechanical pressure was applied with the aid of a special device allowing application of stresses as large as 200 bar. The change of the birefringence caused by a mechanical field $\sigma_{m}$ is defined by the relation

$\Delta n_{i}\left(\sigma_{m}\right)=k\left(\sigma_{m}\right) \cdot \lambda / d_{i}\left(\sigma_{m}\right)$,

where $\Delta n_{i}$ is the birefringence along the $i$-axis, $k$ the order of the interference maximum, $\lambda$ the light wavelength, and $d_{i}$ the sample thickness along the light propagation direction. Under the condition of simultaneously acting thermal and mechanical fields, the birefringence is determined as follows:

$\Delta n_{i}(T, \sigma)=k(T, \sigma) \cdot \lambda / d_{i}(T, \sigma)$

Changing one of the parameters ( $T$ or $\sigma_{m}$ ) and keeping the other one fixed, one can determine the temperature or baric dependence of $\Delta n_{i}$ from the shift of the extremum of the interference pattern.

The combined difference of the piezooptic constants is calculated according to the formula 
$\pi_{i m}^{0}=2 \delta \Delta n_{i} / \sigma_{m}-2 \delta \Delta n_{i} s_{i m}$,

where $\delta \Delta n_{i}$ represents the induced birefringence change for the light propagation direction along the $i$-axis and pressure applied along the $m$-axis. The second term takes into account elastic changes in the sample dimension along the $i$-axis, where $s_{i m}$ is the elastic compliance coefficient.

\section{Results and discussion}

Fig. 1 shows the baric dependence of the birefringence in TGS crystals doped with $5 \%$ L-valine at room temperature and $\lambda=500 \mathrm{~nm}$. As one can see from the figure, the $\Delta n$ value increases linearly up to a stress $\sigma_{m}$ of $\sim 200$ bar. The $\sigma_{x}$ and $\sigma_{y}$ stresses lead to different values and signs of the $\Delta n_{z}$ changes, namely $\delta \Delta n_{z}=$ $-7.2 \cdot 10^{-5}$ (at $\sigma_{y}=200 \mathrm{bar}$ ) and $8.1 \cdot 10^{-5}$ (at $\sigma_{x}=$ 200 bar). The corresponding dependences of $\Delta n_{x}$ and $\Delta n_{\mathrm{y}}$ are quite similar: $\delta \Delta n_{\mathrm{y}}=-6.2 \cdot 10^{-5}$ and $9.8 \cdot 10^{-5}$ for $\sigma_{x}$ and $\sigma_{z}=200 \mathrm{bar}$, and $\delta \Delta n_{\mathrm{x}}=10.1 \cdot 10^{-5}$ and $-12.3 \cdot 10^{-5}$ for $\sigma_{y}$ and $\sigma_{z}=200$ bar. In general, one of the stresses normal to the propagation direction always induces an increase of the birefringence, while the other one has the opposite effect. Furthermore, those increments appear to have different modules ( $\left|\delta \Delta n_{\mathrm{x}}\right|>\left|\delta \Delta n_{\mathrm{y}}\right| \geq\left|\delta \Delta n_{\mathrm{z}}\right|$ for the LTTGS crystals). A similar situation has earlier been observed for a number of $A_{2} B X_{4}$ crystals, as well as for KDP crystals $[16,17]$. It is explained quite well on the basis of the Sellmeier formula even in the simplest case if the baric birefringence changes are supposed to be primarily determined by the density of particles in the crystal. A stress $\sigma_{m}$ leading to an increase of $\Delta n_{\mathrm{i}}$ slows down the spectral change of the baric increment $\delta \Delta n_{i}$, whereas a stress leading to a decrease of $\Delta n_{i}$ accelerates it.

The temperature changes in the principal values of the birefringence for LTTGS are depicted in Fig. 2 for mechanically free and stressed samples.

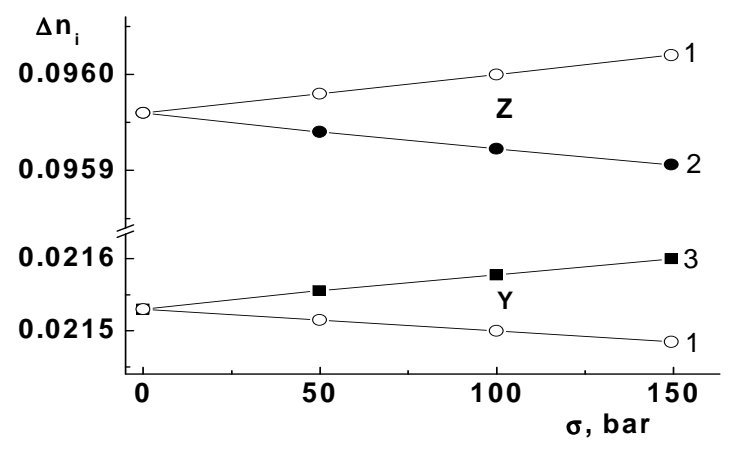

Fig. 1 Baric birefringence changes in a TGS crystal doped with $5 \%$ L-valine, measured at room temperature and $\lambda=500 \mathrm{~nm}: 1-\sigma_{x}$, $2-\sigma_{y}$, and $3-\sigma_{z}$.

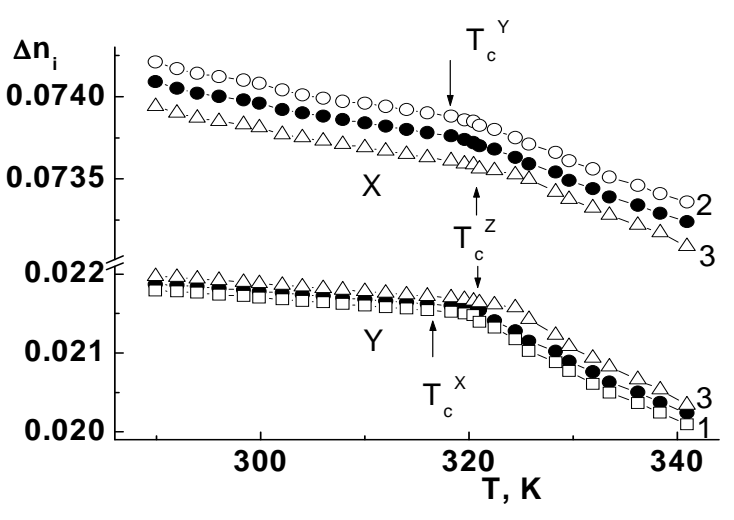

Fig. 2 Temperature dependence of the birefringence in a TGS crystal doped with 5\% L-valine, measured for differently directed uniaxial stresses and $\lambda=500 \mathrm{~nm}: 1-\sigma_{x}, 2-\sigma_{y}$, and $3-\sigma_{z}=200$ bar. Full symbols correspond to a mechanically free crystal.

The general features are as follows: (1) the $\Delta n_{i}$ changes are proportional to the temperature, (2) the derivative $\partial \Delta n_{i} / \partial T$ changes its value while passing through the Curie point, (3) the uniaxial stresses change the absolute value of $\Delta n_{i}$, though the qualitative temperature dependence of $\Delta n_{i}$ remains unchanged, and (4) the PT point is shifted under the influence of the stress. The PT temperature decreases in the case of $\sigma_{x}$ and $\sigma_{y}$ stresses $\left(T_{c}^{X}=320.5 \mathrm{~K}\right.$ and $\left.T_{c}^{Y}=320.2 \mathrm{~K}\right)$, while the $\sigma_{z}$ stress increases the PT point $\left(T_{c}^{Z}=325.2 \mathrm{~K}\right)$. The relevant temperature shift coefficients are equal to $\partial T_{c} / \partial \sigma_{x}=-0.0064, \partial T_{c} / \partial \sigma_{y}=$ -0.0080 and $\partial T_{c} / \partial \sigma_{z}=+0.0171 \mathrm{~K} / \mathrm{bar}$. The "total" (or hydrostatic) coefficient for the PT temperature shift is $\partial T_{c} / \partial p=\sum_{i=1}^{3} \partial T_{c} / \partial \sigma_{i}=+0.0027 \mathrm{~K} /$ bar.

On the one hand, these results correlate well with the data found for pure TGS crystals [18], where the parameters $\partial T_{c} / \partial \sigma_{x}=-7, \partial T_{c} / \partial \sigma_{y}=-8.5$ and $\partial T_{c} / \partial \sigma_{z}=$ $+20 \mathrm{~K} / \mathrm{kbar}$ were derived. The coefficients determined in this work are slightly less, confirming an increase of the elastic stiffness for the doped TGS crystals. On the other hand, analogous results have already been obtained for $\mathrm{LiKSO}_{4}, \mathrm{RbNH}_{4} \mathrm{SO}_{4}$ and $\left(\mathrm{NH}_{4}\right)_{2} \mathrm{BeF}_{4}$ crystals, as well as for the Rochelle salt and KDP $[16,17,19]$, where both increasing and decreasing of $T_{c}$ was observed, depending on the stress direction, and the "total" shift coefficients for $T_{c}$ are negative. The PT shifts were explained by the influence of temperature and stress on the shifts of structural elements taking place at the PT's. Slight discrepancies may be caused by the complex structure of the TGS crystals and possible irregularities on introducing the valine admixture into the crystal structure. When the crystal doped with L-valine is growing, the admixture 
enters into the structure by substituting for one of the glycines. The glycines I-III are planar in the paraelectric phase, but glycine I becomes nonplanar in the ferroelectric phase. When an L-valine molecule substitutes for glycine I, the spontaneous polarization $P_{s}$ becomes fixed and a biasing field appears, altering all the dielectric parameters of the crystals and shifting the PT point. Replacement of glycine by L-valine is more probable if the crystal is growing in the ferroelectric phase. Moreover, a notable shift of the PT point towards higher temperatures is apparently caused by the influence of uniaxial stresses on the ferroelectric domains. In the case of TGS crystals, the latter form "tubes" of oval, or more frequently lenslike, section, stretched along the direction perpendicular to the $c$-axis. The lower values of the baric coefficients for LVTGS, when compared with those of the pure crystal, are associated with the fact that the domains in the doped crystals have large sizes, though irregular shapes and smeared borders, and the concentration of domains decreases in the course of spontaneous aging of the samples.

The above results are in good agreement with the data obtained when studying the influence of uniaxial stress on the hysteresis loop parameters in pure TGS crystals [20]. It was found that the $\sigma_{11}$ and $\sigma_{22}$ stresses induce an increase of the spontaneous polarization and the coercive field, while the $\sigma_{33}$ stress induces a decrease of both parameters. The authors suggest that TGS crystals are "ferroelastoelectrics" [20] rather than simple ferroelectrics. Applying simultaneously both electric and mechanical fields to such crystals leads to their polarization, i.e. ascertaining of one particular oriented state. The $\sigma_{11}$ and $\sigma_{22}$ stresses would then promote domain motion, whereas the stress $\sigma_{33}$ would impede it. In our case, a light electromagnetic wave plays the role of the electric field.

Fig. 3 represents the temperature dependence of the piezooptic constants $\pi_{i m}^{0}$ for the LVTGS crystals. Most of the piezooptic constants $\pi_{i m}^{0}$ increase swiftly when approaching the PT point from the ferroelectric phase, decrease in a jump-like manner when transiting into the paraelectric phase and then become practically temperature independent. The piezooptic constant $\pi_{31}^{0}$ exhibits a somewhat different behaviour: it decreases in the polar phase when approaching $T_{c}$. A comparison of the results obtained here with those obtained for pure TGS crystals [21] reveals the following features. The parameters $\pi_{21}^{0}, \pi_{32}^{0}$ and $\pi_{13}^{0}$ have negative values. This corresponds to a decrease of the $\Delta n_{y}, \Delta n_{z}$ and $\Delta n_{x}$ birefringences under the influence of the stresses $\sigma_{x}, \sigma_{y}$ and $\sigma_{z}$, respectively. Though the absolute values of $\pi_{i m}^{0}$ for the doped crystals are, in general, somewhat larger than for the pure crystal at $T<320 \mathrm{~K}\left(\sim 1-3 \cdot 10^{-12} \mathrm{~m}^{2} / \mathrm{N}\right)$, the temperature slope of the piezooptic parameters essentially decreases while approaching the PT point. The values of the jump-like changes of the piezooptic constants at the PT are noticeably less for the doped crystals than for pure crystals, e.g. $\pi_{13}^{0}=3.4 \cdot 10^{-12}$ and $19.3 \cdot 10^{-12} \mathrm{~m}^{2} / \mathrm{N}$ for LVTGS and TGS, respectively.

The piezooptic effect in crystals of the TGS group is determined by a "true" piezooptic contribution and secondary electrooptics $\left(d \Delta n_{i} / d P_{c}\right)$. The reason for the electrooptic contribution is that, owing to the dependence of the spontaneous polarization on the temperature and the shift of the $P_{s}(T)$ function along the temperature axis under the uniaxial stress $\sigma_{m}$, the spontaneous polarization will change by the value $\delta P_{s}$, and this, in turn, will produce an additional electrooptic change of the birefringence:

$$
\pi_{i m}^{*}=2 \frac{d \Delta n_{i}}{d \sigma_{m}}=2\left[\left(\frac{d \Delta n_{i}}{d \sigma_{m}}\right)_{\text {true }}+\frac{d \Delta n_{i}}{d P_{c}} \cdot \frac{d P_{c}}{d \sigma_{m}}\right],
$$

where $\left(d \Delta n_{i} / d \sigma_{m}\right)_{\text {true }}$ is the contribution of the "true" piezooptic effect to the combined difference $\pi_{i m}^{*}$ and, since

$\pi_{i m}^{0}=\pi_{i m}^{*}+2 \Delta n_{i} s_{i m}$,

the above contribution will equally refer to $\pi_{i m}^{0}$.

It has been shown that the electrooptic effect contributes mostly to the piezooptic constants $\pi_{23}$ and $\pi_{21}$ in the vicinity of the PT. The electrooptic contribution becomes negligible $(\sim 10 \ldots 15 \%)$ far away from the PT point, i.e. inside the ferroelectric phase. These data agree well with the difference observed between the value of $\pi_{23}^{0}$ (or $\pi_{21}^{0}$ ) in the ferroelectric phase and the corresponding value extrapolated from the paraelectric phase.

A comparison of pure and doped TGS crystals shows that the jump-like changes of the piezooptic constants at the PT (i.e. the discontinuity in the $\pi_{i m}^{0}(T)$ function) is conspicuously less in the case of the doped crystals. The reason is in the lower value of the multiplier $d P_{c} / d \sigma_{m}$, which is proportional to $\delta P_{s}$, for the latter crystals, because of smaller $\partial T_{c} / \partial \sigma_{m}$ coefficients and reduced spontaneous polarization.

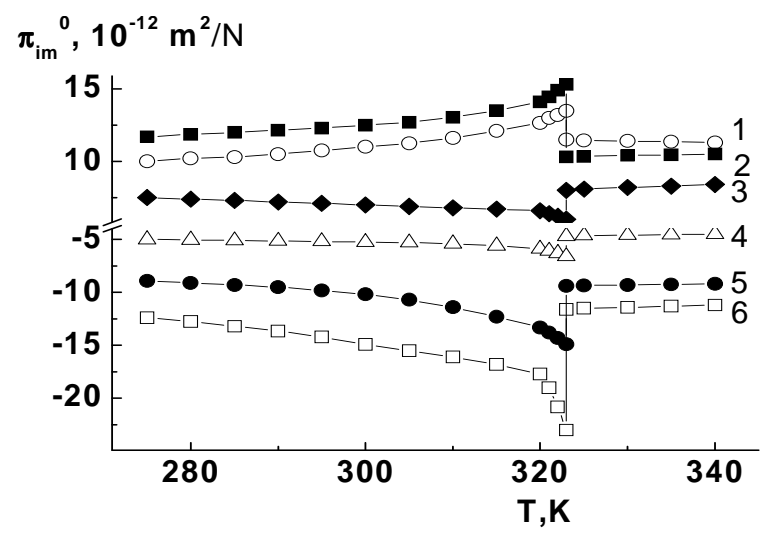

Fig. 3 Temperature dependence of the piezooptic constants of TGS crystals doped with $5 \%$ L-valine, measured at $\lambda=500 \mathrm{~nm}$ : $1-\pi_{23}^{0}, 2-\pi_{12}^{0}, 3-\pi_{31}^{0}, 4-\pi_{21}^{0}, 5-\pi_{32}^{0}$, and $6-\pi_{13}^{0}$. 


\section{Conclusions}

In this work we have studied the influence of uniaxial mechanical stress applied along the crystal-physical axes on the principal birefringence values of TGS crystals doped with $5 \% \mathrm{~L}$-valine. It was found that $\Delta n_{i}$ is sensitive to the action of uniaxial stress. One of the pressures normal to the light propagation direction gives always an increase in $\Delta n_{i}$, while the other one leads to a decrease. In addition, the corresponding increments have different modules, which fulfil the condition $\left|\delta \Delta n_{\mathrm{x}}\right|>\left|\delta \Delta n_{\mathrm{y}}\right| \geq\left|\delta \Delta n_{\mathrm{z}}\right|$. The baric coefficients $\partial T_{c} / \partial \sigma_{m}$ of the PT shift were determined. They are slightly lower than those of pure TGS crystals. This indicates that the stiffness of TGS crystals should increase when admixtures are introduced. The "total" (hydrostatic) coefficient $\partial T_{c} / \partial p=\sum_{i=1}^{3} \partial T_{c} / \partial \sigma_{i}$ of the PT shift under uniaxial stress was estimated.

It was established that the absolute values of $\pi_{i m}^{0}$ of the doped crystals are somewhat larger than those of the pure crystals at $T<320 \mathrm{~K}$ and the temperature slope of $\pi_{i m}^{0}$ decreases notably near the PT point. The values of the jump-like changes occurring in all of the piezooptic constants at the PT are essentially lower for the doped crystals than for the pure ones.

\section{References}

[1] K.L. Bye, P.W. Whips, E.T. Keve, Ferroelectrics 4 (1972) 235.

[2] M. Koralewski, T. Stankowska, T. Jasinski, Jpn. J. Appl. Phys. 26 (1987) 383.

[3] B. Brezina, M. Havrankova, Cryst. Res. Technol. 20 (1985) 787.
[4] B. Brezina, M. Havrankova, Cryst. Res. Technol. 20 (1985) 781.

[5] J. Stankowska, A. Czarnecka, Ferroelectrics 98 (1989) 95.

[6] A. Czarnecka, J. Stankowska, S. Mielcarek, Acta Phys. Pol. A 85 (1994) 849.

[7] J. Stankowska, A. Czarnecka, S. Mielcarek, Ferroelectrics 172 (1995) 221.

[8] J. Stankowska, A. Czarnecka, G. Kwitkowska, Ferroelectrics 108 (1990) 325.

[9] V.Y. Stadnyk, M.O. Romanyuk, Ferroelectrics 317 (2005) 287.

[10] V.Y. Stadnyk, Yu.I. Kiryk, I.M. Matviishyn, Ukr. Fiz. Zh. 55 (2010) 431.

[11] M.O. Romanyuk, B.G. Mytsyk, M. Varikash, Izv. Akad. Nauk BSSR, Ser. Fiz.-Mat. Nauk 6 (1980) 105 (in Russian).

[12] B.G. Mytsyk, M.O. Romanyuk, Ukr. Fiz. Zh. 28 (1983) 538 (in Russian).

[13] M.O. Romanyuk, B.G. Mytsyk, Ukr. Fiz. Zh. 27 (1982) 1206 (in Russian).

[14] M.O. Romanyuk, B.G. Mytsyk, V.M. Varikash, Fiz. Tverd. Tela 25 (1983) 1670 (in Russian).

[15] B.G. Mytsyk, M.O. Romanyuk, Izv. Akad. Nauk SSSR, Ser. Fiz. 47 (1983) 674 (in Russian).

[16] M.O. Romanyuk, V.Y. Stadnyk, Condens. Matter Phys. 2 (1999) 711.

[17] V.Y. Stadnyk, O.S. Kushnir, R.S. Brezvin, V.M. Gaba, Opt. Spectrosc. 106 (2009) 614.

[18] K. Imai, J. Phys. Soc. Jpn. 36 (1974) 1069.

[19] V.Y. Stadnyk, M.O. Romanyuk, Ferroelectrics 317 (2005) 255.

[20] E.A. Kafadarova, I.N. Polandov, V.M. Petrov, A.N. Popov, Fiz. Tverd. Tela 21 (1979) 620 (in Russian).

[21] E.F. Dudnik, V.M. Duda, A.I. Kushnaryov, Fiz. Tverd. Tela 42 (2000) 133 (in Russian).

Proceeding of the XVI International Seminar on Physics and Chemistry of Solids, Lviv, June 6-9, 2010. 